\title{
Muon conversion to an electron in nuclei in the minimal R-symmetric supersymmetric standard model
}

\author{
Ke-Sheng Sun $\oplus^{1, *}$ Sheng-Kai Cui, ${ }^{2,3, \dagger}$ Wei Li, ${ }^{2,3, \$}$ and Hai-Bin Zhang $\circledast^{2,3, \S}$ \\ ${ }^{1}$ Department of Physics, Baoding University, Baoding 071000,China \\ ${ }^{2}$ Department of Physics, Hebei University, Baoding 071002, China \\ ${ }^{3}$ Key Laboratory of High-Precision Computation and Application of Quantum Field \\ Theory of Hebei Province, Baoding 071002, China
}

(Received 27 April 2020; accepted 7 August 2020; published 28 August 2020)

\begin{abstract}
We analyze the lepton flavor violating process $\mu$-e conversion in the framework of the minimal R-symmetric supersymmetric standard model. The theoretical predictions are determined by considering the experimental constraint on parameter $\delta^{12}$ from the lepton flavor violating decay $\mu \rightarrow e \gamma$. The predictions for $\mathrm{CR}\left(\mu-e\right.$, nucleus) in nuclei are not sensitive to $\tan \beta$ or $m_{A}$ and take values in a narrow region. The numerical results show that $\gamma$ penguins dominate the predictions on $\mathrm{CR}(\mu-e$, nucleus), and the contribution from Higgs penguins is insignificant. The $Z$ penguins and box diagrams are less dominant in the predictions on $\mathrm{CR}(\mu-e$, nucleus $)$ in a large parameter region. For small squark mass parameter, the contribution from box diagrams is comparable with dipole contribution from $\gamma$ penguins. The theoretical predictions on conversion rate $\mathrm{CR}(\mu-e$, nucleus $)$ in an $\mathrm{Al}$ or Ti target can be enhanced close to the future experimental sensitivities and are very promising to be observed in near future experiments.
\end{abstract}

DOI: 10.1103/PhysRevD.102.035029

\section{INTRODUCTION}

Searching for lepton flavor violating (LFV) decays are of great importance in probing new physics (NP) beyond the Standard Model (SM) in which the theoretical predictions on those LFV decays are suppressed by small masses of neutrinos and far beyond the experimental accessibility. There are many different ways to search LFV such as $\mu \rightarrow e \gamma, \mu \rightarrow 3 e, \mu-e$ conversion in nucleus, $\tau$ decays, hadron decays, and so on. However, no LFV signals have been observed in experiment up to now. The $\mu-e$ conversion in nucleus is a process that muons are captured in a target of atomic nucleus and form a muonic atom. Several experiments have been built or planned to be built to search for this process. Current limit on the $\mu-e$ conversion rate is $4.6 \times 10^{-12}$ for a Ti target at TRIUMF [1], $4.3 \times 10^{-12}$ for a Ti target, and $7 \times 10^{-13}$ for a Au target at SINDRUM-II experiment [2]. In the future, this LFV process may be observed by experiments with improved sensitivity. A future prospects of $10^{-13}$ for a $\mathrm{C}$ target or $10^{-14}$ for a $\mathrm{SiC}$ target

\footnotetext{
*sunkesheng@126.com; sunkesheng@bdu.edu.cn

2252953633@qq.com

*watliwei@163.com

§hbzhang@hbu.edu.cn
}

Published by the American Physical Society under the terms of the Creative Commons Attribution 4.0 International license. Further distribution of this work must maintain attribution to the author(s) and the published article's title, journal citation, and DOI. Funded by SCOAP. at DeeMe [3], $10^{-18}$ for a Ti target at PRISM [4], and $10^{-16}-10^{-17}$ for an $\mathrm{Al}$ target at Mu2e and COMET $[5,6]$ will be achieved, which will improve the current experimental limits by several orders of magnitude.

The $\mu$-e conversion rate has been calculated in the literature for various extensions of the SM. Some seesaw models with right-handed neutrinos [7-13], scalar triplets [14-16], fermion singlet [17], and fermion triplets [18] can have $\mathrm{CR}(\mu-e$, nucleus) close to the experimental sensitivity. There are a few studies within models of nonsupersymmetry (SUSY), such as unparticle model [19,20], littlest Higgs model [21,22], left-right symmetric models [23], 331 model [24], and so on. There are also a few studies within models of SUSY, such as the minimal supersymmetric Standard Model (MSSM) [25], R-parity violating SUSY [26], low-scale seesaw models of minimal supergravity [27], a minimal supersymmetric extension of the SM with local gauged B and L [28,29], the constrained minimal supersymmetric Standard Model-seesaw [30], $\mu \nu$ SSM [31], and so on. $\mu$ - $e$ conversion is particularly sensitive to Higgs mediated LFV because it is not suppressed by small Yukawa couplings as $\mu \rightarrow e \gamma$ and $\mu \rightarrow 3 e$, and Higgs-induced LFV occurs in many NP models [32]. Some pedagogical introductions on the theoretical motivations for charged LFV and the experimental aspects are provided in Ref. [33-35].

In this paper, we will study the LFV process $\mu$-e conversion in the minimal R-symmetric supersymmetric standard model (MRSSM) [36]. The MRSSM has an unbroken global $U(1)_{R}$ symmetry and provides a new solution to the supersymmetric flavor problem in MSSM. 
In this model, R-symmetry forbids Majorana gaugino masses, $\mu$ term, $A$ terms, and all left-right squark and slepton mass mixings. The $R$-charged Higgs $S U(2)_{L}$ doublets $\hat{R}_{u}$ and $\hat{R}_{d}$ are introduced in MRSSM to yield the Dirac mass terms of higgsinos. Additional superfields $\hat{S}, \hat{T}$, and $\hat{O}$ are introduced to yield Dirac mass terms of gauginos. Studies on phenomenology in the MRSSM can be found in the literature [37-55]. Similar to MSSM, the off-diagonal entries $\delta^{i j}$ in slepton mass matrices $m_{l}^{2}$ and $m_{r}^{2}$ dominate the LFV process $\mu-e$ conversion. Taking account of the constraints from radiative decays $\mu \rightarrow e \gamma$ on the off-diagonal parameters $\delta^{i j}$, we explore the $\mu$-e conversion rate as a function of off-diagonal parameter $\delta^{i j}$ and other model parameters.

The paper is organized as follows. In Sec. II, we present the details of the MRSSM. All relevant mass matrices and mixing matrices are provided. Feynman diagrams contributing to $\mu-e$ conversion in MRSSM are given at one loop level. The $\mu-e$ conversion rate is computed in the effective Lagrangian method, and notations and conventions for effective operators and Wilson coefficients are also listed. The numerical results are presented in Sec. III, and the conclusion is drawn in Sec. IV.

\section{MRSSM}

In this section, we firstly provide a simple overview of the MRSSM in order to fix the notations we use in this paper. The MRSSM has the same gauge symmetry $S U(3)_{C} \times S U(2)_{L} \times U(1)_{Y}$ as the SM and MSSM. The spectrum of fields in the MRSSM contains the standard MSSM matter, Higgs, and gauge superfields augmented by chiral adjoints $\hat{\mathcal{O}}, \hat{T}, \hat{S}$ and two $R$-Higgs iso-doublets. The general form of the superpotential of the MRSSM is given by [37]

$$
\begin{aligned}
\mathcal{W}_{\mathrm{MRSSM}}= & \mu_{d}\left(\hat{R}_{d} \hat{H}_{d}\right)+\mu_{u}\left(\hat{R}_{u} \hat{H}_{u}\right)+\Lambda_{d}\left(\hat{R}_{d} \hat{T}\right) \hat{H}_{d} \\
& +\Lambda_{u}\left(\hat{R}_{u} \hat{T}\right) \hat{H}_{u}+\lambda_{d} \hat{S}\left(\hat{R}_{d} \hat{H}_{d}\right)+\lambda_{u} \hat{S}\left(\hat{R}_{u} \hat{H}_{u}\right) \\
& -Y_{d} \hat{d}\left(\hat{q} \hat{H}_{d}\right)-Y_{e} \hat{e}\left(\hat{l} \hat{H}_{d}\right)+Y_{u} \hat{u}\left(\hat{q} \hat{H}_{u}\right),
\end{aligned}
$$

where $\hat{H}_{u}$ and $\hat{H}_{d}$ are the MSSM-like Higgs weak isodoublets, $\hat{R}_{u}$ and $\hat{R}_{d}$ are the $R$-charged Higgs $S U(2)_{L}$ doublets, and the corresponding Dirac higgsino mass parameters are denoted as $\mu_{u}$ and $\mu_{d}$. Although R-symmetry forbids the $\mu$ terms of the MSSM, the bilinear combinations of the normal Higgs $S U(2)_{L}$ doublets $\hat{H}_{u}$ and $\hat{H}_{d}$ with the Higgs $S U(2)_{L}$ doublets $\hat{R}_{u}$ and $\hat{R}_{d}$ are allowed in Eq. (1). Parameters $\lambda_{u}, \lambda_{d}, \Lambda_{u}$, and $\Lambda_{d}$ are Yukawa-like trilinear terms involving the singlet $\hat{S}$ and the triplet $\hat{T}$. For our phenomenological studies we take the soft-breaking terms involving scalar mass that have been considered in [39]

$$
\begin{aligned}
V_{S B, S}= & m_{H_{d}}^{2}\left(\left|H_{d}^{0}\right|^{2}+\left|H_{d}^{-}\right|^{2}\right)+m_{H_{u}}^{2}\left(\left|H_{u}^{0}\right|^{2}+\left|H_{u}^{+}\right|^{2}\right)+\left(B_{\mu}\left(H_{d}^{-} H_{u}^{+}-H_{d}^{0} H_{u}^{0}\right)+\text { H.c. }\right) \\
& +m_{R_{d}}^{2}\left(\left|R_{d}^{0}\right|^{2}+\left|R_{d}^{+}\right|^{2}\right)+m_{R_{u}}^{2}\left(\left|R_{u}^{0}\right|^{2}+\left|R_{u}^{-}\right|^{2}\right)+m_{T}^{2}\left(\left|T^{0}\right|^{2}+\left|T^{-}\right|^{2}+\left|T^{+}\right|^{2}\right) \\
& +m_{S}^{2}|S|^{2}+m_{O}^{2}\left|O^{2}\right|+\tilde{d}_{L, i}^{*} m_{q, i j}^{2} \tilde{d}_{L, j}+\tilde{d}_{R, i}^{*} m_{d, i j}^{2} \tilde{d}_{R, j}+\tilde{u}_{L, i}^{*} m_{q, i j}^{2} \tilde{u}_{L, j} \\
& +\tilde{u}_{R, i}^{*} m_{u, i j}^{2} \tilde{u}_{R, j}+\tilde{e}_{L, i}^{*} m_{l, i j}^{2} \tilde{e}_{L, j}+\tilde{e}_{R, i}^{*} m_{r, i j}^{2} \tilde{e}_{R, j}+\tilde{\nu}_{L, i}^{*} m_{l, i j}^{2} \tilde{\nu}_{L, j} .
\end{aligned}
$$

All trilinear scalar couplings involving Higgs bosons to squarks and sleptons are forbidden in Eq. (2) because the sfermions have an R-charge and these terms are non- $\mathrm{R}$ invariant, and this relaxes the flavor problem of the MSSM [36]. The Dirac nature is a manifest feature of MRSSM fermions and the soft-breaking Dirac mass terms of the singlet $\hat{S}$, triplet $\hat{T}$, and octet $\hat{O}$ take the form as

$$
V_{S B, D G}=M_{D}^{B} \tilde{B} \tilde{S}+M_{D}^{W} \tilde{W}^{a} \tilde{T}^{a}+M_{D}^{O} \tilde{g} \tilde{O}+\text { H.c. },
$$

where $\tilde{B}, \tilde{W}$, and $\tilde{g}$ are usually MSSM Weyl fermions. R-Higgs bosons do not develop vacuum expectation values since they carry R-charge 2. After electroweak symmetry breaking the singlet and triplet vacuum expectation values effectively modify the $\mu_{u}$ and $\mu_{d}$, and the modified $\mu_{i}$ parameters are given by

$$
\begin{aligned}
& \mu_{d}^{\mathrm{eff},+}=\frac{1}{2} \Lambda_{d} v_{T}+\frac{1}{\sqrt{2}} \lambda_{d} v_{S}+\mu_{d}, \\
& \mu_{u}^{\mathrm{eff},-}=-\frac{1}{2} \Lambda_{u} v_{T}+\frac{1}{\sqrt{2}} \lambda_{u} v_{S}+\mu_{u} .
\end{aligned}
$$

The $v_{T}$ and $v_{S}$ are vacuum expectation values of $\hat{T}$ and $\hat{S}$ which carry R-charge zero.

In the weak basis $\left(\sigma_{d}, \sigma_{u}, \sigma_{S}, \sigma_{T}\right)$, the pseudoscalar Higgs boson mass matrix and the diagonalization procedure are

$$
\begin{aligned}
& \mathcal{M}_{A^{0}}=\left(\begin{array}{cccc}
B_{\mu} \frac{v_{u}}{v_{d}} & B_{\mu} & 0 & 0 \\
B_{\mu} & B_{\mu} \frac{v_{d}}{v_{u}} & 0 & 0 \\
0 & 0 & m_{S}^{2}+\frac{\lambda_{d}^{2} v_{d}^{2}+\lambda_{u}^{2} v_{u}^{2}}{2} & \frac{\lambda_{d} \Lambda_{d} v_{d}^{2}-\lambda_{u} \Lambda_{u} v_{u}^{2}}{2 \sqrt{2}} \\
0 & 0 & \frac{\lambda_{d} \Lambda_{d} v_{d}^{2}-\lambda_{u} \Lambda_{u} v_{u}^{2}}{2 \sqrt{2}} & m_{T}^{2}+\frac{\Lambda_{d}^{2} v_{d}^{2}+\Lambda_{u}^{2} v_{u}^{2}}{4}
\end{array}\right), \\
& Z^{A} \mathcal{M}_{A^{0}}\left(Z^{A}\right)^{\dagger}=\mathcal{M}_{A^{0}}^{\text {diag }} .
\end{aligned}
$$


In the weak basis $\left(\phi_{d}, \phi_{u}, \phi_{S}, \phi_{T}\right)$, the scalar Higgs boson mass matrix and the diagonalization procedure are

$$
\mathcal{M}_{h}=\left(\begin{array}{ll}
\mathcal{M}_{11} & \mathcal{M}_{21}^{T} \\
\mathcal{M}_{21} & \mathcal{M}_{22}
\end{array}\right), \quad Z^{h} \mathcal{M}_{h}\left(Z^{h}\right)^{\dagger}=\mathcal{M}_{h}^{\text {diag }},
$$

where the submatrices $\left(c_{\beta}=\cos \beta, s_{\beta}=\sin \beta\right)$ are

$$
\begin{aligned}
& \mathcal{M}_{11}=\left(\begin{array}{cc}
m_{Z}^{2} c_{\beta}^{2}+m_{A}^{2} s_{\beta}^{2} & -\left(m_{Z}^{2}+m_{A}^{2}\right) s_{\beta} c_{\beta} \\
-\left(m_{Z}^{2}+m_{A}^{2}\right) s_{\beta} c_{\beta} & m_{Z}^{2} s_{\beta}^{2}+m_{A}^{2} c_{\beta}^{2}
\end{array}\right), \\
& \mathcal{M}_{21}=\left(\begin{array}{cc}
v_{d}\left(\sqrt{2} \lambda_{d} \mu_{d}^{\mathrm{eff},+}-g_{1} M_{B}^{D}\right) & v_{u}\left(\sqrt{2} \lambda_{u} \mu_{u}^{\mathrm{eff},-}+g_{1} M_{B}^{D}\right) \\
v_{d}\left(\Lambda_{d} \mu_{d}^{\mathrm{eff},+}+g_{2} M_{W}^{D}\right) & -v_{u}\left(\Lambda_{u} \mu_{u}^{\mathrm{eff}, 1}+g_{2} M_{W}^{D}\right)
\end{array}\right), \\
& \mathcal{M}_{22}=\left(\begin{array}{cc}
4\left(M_{B}^{D}\right)^{2}+m_{S}^{2}+\frac{\lambda_{d}^{2} v_{d}^{2}+\lambda_{u}^{2} v_{u}^{2}}{2} & \frac{\lambda_{d} \Lambda_{d} v_{d}^{2}-\lambda_{u} \Lambda_{u} v_{u}^{2}}{2 \sqrt{2}} \\
\frac{\lambda_{d} \Lambda_{d} v_{d}^{2}-\lambda_{u} \Lambda_{u} v_{u}^{2}}{2 \sqrt{2}} & 4\left(M_{W}^{D}\right)^{2}+m_{T}^{2}+\frac{\Lambda_{d}^{2} v_{d}^{2}+\Lambda_{u}^{2} v_{u}^{2}}{4}
\end{array}\right) .
\end{aligned}
$$

The number of neutralino degrees of freedom in MRSSM is doubled compared to MSSM as the neutralinos are Dirac type. In the weak basis of four neutral electroweak two-component fermions $\xi_{i}=\left(\tilde{B}, \tilde{W}^{0}, \tilde{R}_{d}^{0}, \tilde{R}_{u}^{0}\right)$ with R-charge 1 and four neutral electroweak twocomponent fermions $\varsigma_{i}=\left(\tilde{S}, \tilde{T}^{0}, \tilde{H}_{d}^{0}, \tilde{H}_{u}^{0}\right)$ with R-charge -1 , the neutralino mass matrix and the diagonalization procedure are

$$
\begin{aligned}
& m_{\chi^{0}}=\left(\begin{array}{cccc}
M_{D}^{B} & 0 & -\frac{1}{2} g_{1} v_{d} & \frac{1}{2} g_{1} v_{u} \\
0 & M_{D}^{W} & \frac{1}{2} g_{2} v_{d} & -\frac{1}{2} g_{2} v_{u} \\
-\frac{1}{\sqrt{2}} \lambda_{d} v_{d} & -\frac{1}{2} \Lambda_{d} v_{d} & -\mu_{d}^{\mathrm{eff},+} & 0 \\
\frac{1}{\sqrt{2}} \lambda_{u} v_{u} & -\frac{1}{2} \Lambda_{u} v_{u} & 0 & \mu_{u}^{\mathrm{eff},-}
\end{array}\right), \\
& \left(N^{1}\right)^{*} m_{\chi^{0}}\left(N^{2}\right)^{\dagger}=m_{\chi^{0}}^{\mathrm{diag}} .
\end{aligned}
$$

The mass eigenstates $\kappa_{i}$ and $\varphi_{i}$, and physical fourcomponent Dirac neutralinos are

$$
\xi_{i}=\sum_{j=1}^{4}\left(N_{j i}^{1}\right)^{*} \kappa_{j}, \quad \varsigma_{i}=\sum_{j=1}^{4}\left(N_{i j}^{2}\right)^{*} \varphi_{j}, \quad \chi_{i}^{0}=\left(\begin{array}{c}
\kappa_{i} \\
\varphi_{i}^{*}
\end{array}\right) .
$$

The number of chargino degrees of freedom in the MRSSM is also doubled compared to MSSM and these charginos can be grouped to two separated chargino sectors according to their R-charge. The $\chi^{ \pm}$-charginos sector has R-charge 1 electric charge; the $\rho$-charginos sector has R-charge -1 electric charge. In the basis $\xi_{i}^{+}=\left(\tilde{W}^{+}, \tilde{R}_{d}^{+}\right)$ and $\varsigma_{i}^{-}=\left(\tilde{T}^{-}, \tilde{H}_{d}^{-}\right)$, the $\chi^{ \pm}$-charginos mass matrix and the diagonalization procedure are

$$
\begin{aligned}
& m_{\chi^{ \pm}}=\left(\begin{array}{cc}
g_{2} v_{T}+M_{D}^{W} & \frac{1}{\sqrt{2}} \Lambda_{d} v_{d} \\
\frac{1}{\sqrt{2}} g_{2} v_{d} & -\frac{1}{2} \Lambda_{d} v_{T}+\frac{1}{\sqrt{2}} \lambda_{d} v_{S}+\mu_{d}
\end{array}\right), \\
& \left(U^{1}\right)^{*} m_{\chi^{ \pm}}\left(V^{1}\right)^{\dagger}=m_{\chi^{ \pm}}^{\text {diag }} .
\end{aligned}
$$

The mass eigenstates $\lambda_{i}^{ \pm}$and physical four-component Dirac charginos are

$$
\xi_{i}^{+}=\sum_{j=1}^{2}\left(V_{i j}^{1}\right)^{*} \lambda_{j}^{+}, \quad \varsigma_{i}^{-}=\sum_{j=1}^{2}\left(U_{j i}^{1}\right)^{*} \lambda_{j}^{-}, \quad \chi_{i}^{ \pm}=\left(\begin{array}{c}
\lambda_{i}^{+} \\
\lambda_{i}^{-*}
\end{array}\right) .
$$

Here, we do not discuss the $\rho$-charginos sector in detail since it does not contribute to $\mu$-e conversion. More information about the $\rho$-charginos can be found in Refs. $[39,41,43,53]$.

In MRSSM the LFV decays mainly originate from the potential misalignment in sleptons mass matrices. In the gauge eigenstate basis $\tilde{\nu}_{i L}$, the sneutrino mass matrix and the diagonalization procedure are

$m_{\tilde{\nu}}^{2}=m_{l}^{2}+\frac{1}{8}\left(g_{1}^{2}+g_{2}^{2}\right)\left(v_{d}^{2}-v_{u}^{2}\right)+g_{2} v_{T} M_{D}^{W}-g_{1} v_{S} M_{D}^{B}$,

$Z^{V} m_{\tilde{\nu}}^{2}\left(Z^{V}\right)^{\dagger}=m_{\tilde{\nu}}^{2, \text { diag }}$,

where the last two terms in mass matrix are newly introduced by MRSSM. The slepton mass matrix and the diagonalization procedure are

$$
\begin{aligned}
m_{\tilde{e}}^{2}= & \left(\begin{array}{cc}
\left(m_{\tilde{e}}^{2}\right)_{L L} & 0 \\
0 & \left(m_{\tilde{e}}^{2}\right)_{R R}
\end{array}\right), \quad Z^{E} m_{\tilde{e}}^{2}\left(Z^{E}\right)^{\dagger}=m_{\tilde{e}}^{2, \text { diag }}, \\
\left(m_{\tilde{e}}^{2}\right)_{L L}= & m_{l}^{2}+\frac{1}{2} v_{d}^{2}\left|Y_{e}\right|^{2}+\frac{1}{8}\left(g_{1}^{2}-g_{2}^{2}\right)\left(v_{d}^{2}-v_{u}^{2}\right) \\
& -g_{1} v_{S} M_{D}^{B}-g_{2} v_{T} M_{D}^{W}, \\
\left(m_{\tilde{e}}^{2}\right)_{R R}= & m_{r}^{2}+\frac{1}{2} v_{d}^{2}\left|Y_{e}\right|^{2}+\frac{1}{4} g_{1}^{2}\left(v_{u}^{2}-v_{d}^{2}\right)+2 g_{1} v_{S} M_{D}^{B} .
\end{aligned}
$$


The sources of LFV are the off-diagonal entries of the $3 \times 3$ soft supersymmetry breaking matrices $m_{l}^{2}$ and $m_{r}^{2}$ in Eqs. (8) and (9). From Eq. (9) we can see that the left-right slepton mass mixing is absent in the MRSSM, whereas the $A$ terms are present in the MSSM.

The mass matrix for up squarks and down squarks, and the relevant diagonalization procedure are

$$
\begin{aligned}
m_{\tilde{u}}^{2}= & \left(\begin{array}{cc}
\left(m_{\tilde{u}}^{2}\right)_{L L} & 0 \\
0 & \left(m_{\tilde{u}}^{2}\right)_{R R}
\end{array}\right), \quad Z^{U} m_{\tilde{u}}^{2}\left(Z^{U}\right)^{\dagger}=m_{\tilde{u}}^{2, \text { diag }}, \\
m_{\tilde{d}}^{2}= & \left(\begin{array}{cc}
\left(m_{\tilde{d}}^{2}\right)_{L L} & 0 \\
0 & \left(m_{\tilde{d}}^{2}\right)_{R R}
\end{array}\right), \quad Z^{D} m_{\tilde{d}}^{2}\left(Z^{D}\right)^{\dagger}=m_{\tilde{d}}^{2, \text { diag }}, \\
\left(m_{\tilde{u}}^{2}\right)_{L L}= & m_{\tilde{q}}^{2}+\frac{1}{2} v_{u}^{2}\left|Y_{u}\right|^{2}+\frac{1}{24}\left(g_{1}^{2}-3 g_{2}^{2}\right)\left(v_{u}^{2}-v_{d}^{2}\right) \\
& +\frac{1}{3} g_{1} v_{S} M_{D}^{B}+g_{2} v_{T} M_{D}^{W}, \\
\left(m_{\tilde{u}}^{2}\right)_{R R}= & m_{\tilde{u}}^{2}+\frac{1}{2} v_{u}^{2}\left|Y_{u}\right|^{2}+\frac{1}{6} g_{1}^{2}\left(v_{d}^{2}-v_{u}^{2}\right)-\frac{4}{3} g_{1} v_{S} M_{D}^{B}, \\
\left(m_{\tilde{d}}^{2}\right)_{L L}= & m_{\tilde{q}}^{2}+\frac{1}{2} v_{d}^{2}\left|Y_{d}\right|^{2}+\frac{1}{24}\left(g_{1}^{2}+3 g_{2}^{2}\right)\left(v_{u}^{2}-v_{d}^{2}\right) \\
& +\frac{1}{3} g_{1} v_{S} M_{D}^{B}-g_{2} v_{T} M_{D}^{W}, \\
\left(m_{\tilde{d}}^{2}\right)_{R R}= & m_{\tilde{d}}^{2}+\frac{1}{2} v_{d}^{2}\left|Y_{d}\right|^{2}+\frac{1}{12} g_{1}^{2}\left(v_{u}^{2}-v_{d}^{2}\right)+\frac{2}{3} g_{1} v_{S} M_{D}^{B} .
\end{aligned}
$$

The MRSSM has been implemented in the Mathematica package SARAH [56-58], and we use the Feynman rules generated with SARAH-4.14.3 in our work. In the MRSSM, violating of lepton flavor arises at the one-loop level. In the MRSSM, $\mu-e$ conversion is induced by the Feynman diagrams given in Fig. 1. The various contributions to this process can be classified into Higgs penguins, $\gamma$ penguins, $Z$ penguins, and box diagrams. In the effective Lagrangian method, one can derive the effective Lagrangian relevant for $\mu$-e conversion as [59]

$$
\begin{aligned}
\mathcal{L}_{\text {eff }}= & e \bar{l}_{e} \gamma^{\mu}\left(K_{1}^{L} P_{L}+K_{1}^{R} P_{R}\right) l_{\mu} A_{\mu} \\
& +\sum_{K=S, V}^{X, Y=L, R} B_{X Y}^{K} \bar{l}_{e} \Gamma_{K} P_{X} l_{\mu} \bar{d} \Gamma_{K} P_{Y} d \\
& +\sum_{K=S, V}^{X, Y=L, R} C_{X Y}^{K} \bar{l}_{e} \Gamma_{K} P_{X} l_{\mu} \bar{u} \Gamma_{K} P_{Y} u+\text { H.c. }
\end{aligned}
$$

The conversion rate $\mathrm{CR}(\mu-e$, nucleus) in nuclei can be calculated by

$$
\begin{aligned}
& \mathrm{CR}(\mu-e, \text { nucleus }) \\
& =\sum_{X=L, R} \frac{p_{e} E_{e} m_{\mu}^{3} G_{F}^{2} \alpha^{3} Z_{\mathrm{eff}}^{4} F_{p}^{2}}{8 \pi^{2} Z \Gamma_{\mathrm{capt}}} \\
& \quad \times\left|(Z+N)\left(g_{X V}^{(0)}+g_{X S}^{(0)}\right)+(Z-N)\left(g_{X V}^{(1)}+g_{X S}^{(1)}\right)\right|^{2} .
\end{aligned}
$$

Here $p_{e}$ and $E_{e}\left(\sim m_{\mu}\right.$ in the numerical evaluation) are the momentum and energy of the electron. $G_{F}$ and $\alpha$ are the Fermi constant and the fine structure constant, respectively. $Z_{\text {eff }}$ is the effective atomic charge. $Z$ and $N$ are the number of protons and neutrons in the nucleus. $F_{p}$ is the nuclear form factor, and $\Gamma_{\text {capt }}$ is the total muon capture rate. The values of $Z_{\text {eff }}, F_{p}$ and $\Gamma_{\text {capt }}$ that will be used in the phenomenological analysis below are given in Table. I. At the quark level, the $g_{X K}^{(i)}$ factors (with $i=0,1, X=L, R$, and $K=S, V$ ) can be written as combinations of effective couplings

$$
g_{X K}^{(i)}=\frac{1}{2} \sum_{q=u, d, s}\left(g_{X K(q)} G_{K}^{(q, p)}+(-1)^{i} g_{X K(q)} G_{K}^{(q, n)}\right) .
$$

The values of $G_{K}$ factors are $G_{S}^{(u, p)}=G_{S}^{(d, n)}=5.1$, $G_{S}^{(d, p)}=G_{S}^{(u, n)}=4.3, \quad G_{S}^{(s, p)}=G_{S}^{(s, n)}=2.5, \quad G_{V}^{(u, p)}=$ $G_{V}^{(d, n)}=2$, and $G_{V}^{(d, p)}=G_{V}^{(u, n)}=1$. The $g_{X K(q)}$ coefficients can be written as combinations of Wilson coefficients

$$
\begin{aligned}
& g_{L V(q)}=\frac{\sqrt{2}}{G_{F}}\left(e^{2} Q_{q}\left(K_{1}^{L}-K_{2}^{R}\right)-\frac{1}{2}\left(C_{l l q q}^{V L L}+C_{l l q q}^{V L R}\right)\right), \\
& g_{L S(q)}=-\frac{\sqrt{2}}{2 G_{F}}\left(C_{l l q q}^{S L L}+C_{l l q q}^{S L R}\right),
\end{aligned}
$$

where $Q_{q}$ are the electric charge of quarks, $C_{l l q q}^{S L L}$ equals $B_{X Y}^{K}\left(C_{X Y}^{K}\right)$ for d-quarks (u-quarks), $g_{R V(q)}=$ $g_{L V(q)} \mid L \rightarrow R$, and $g_{R S(q)}=g_{L S(q)} \mid L \rightarrow R$.

\section{NUMERICAL ANALYSIS}

We now turn to the numerical analysis of the one-loop corrections to $\mu-e$ conversion in nuclei in the MRSSM by using the full evaluation within the framework of SARAH4.14.3 [56-58] and SPheno-4.0.4 [60,61]. The computation is done in a low scale version of SPheno and all free parameters are given at the SUSY scale. The experimental values of the Higgs mass and $W$ boson mass can impose stringent and nontrivial constraints on the model parameters. The one-loop and leading two-loop corrections to the lightest (SM-like) Higgs boson in the MRSSM have been computed in Ref. [39] and several sets of benchmark points are given. These benchmark points make it possible to accommodate a proper Higgs boson mass of around $125 \mathrm{GeV}$ in MRSSM. The Higgs sector of the benchmark points is checked against existing experimental data using HiggsBounds and HiggsSignals and the Higgs potential of the MRSSM is checked for possible presence of deeper minima in the parameter space. There are also other restrictions. The $W$ boson mass is found in agreement with the experimental value from combined LEP and Tevatron, and low energy $B$ meson physics observables are found in agreement with measurements. All benchmark points are allowed by the fits to electroweak precision 


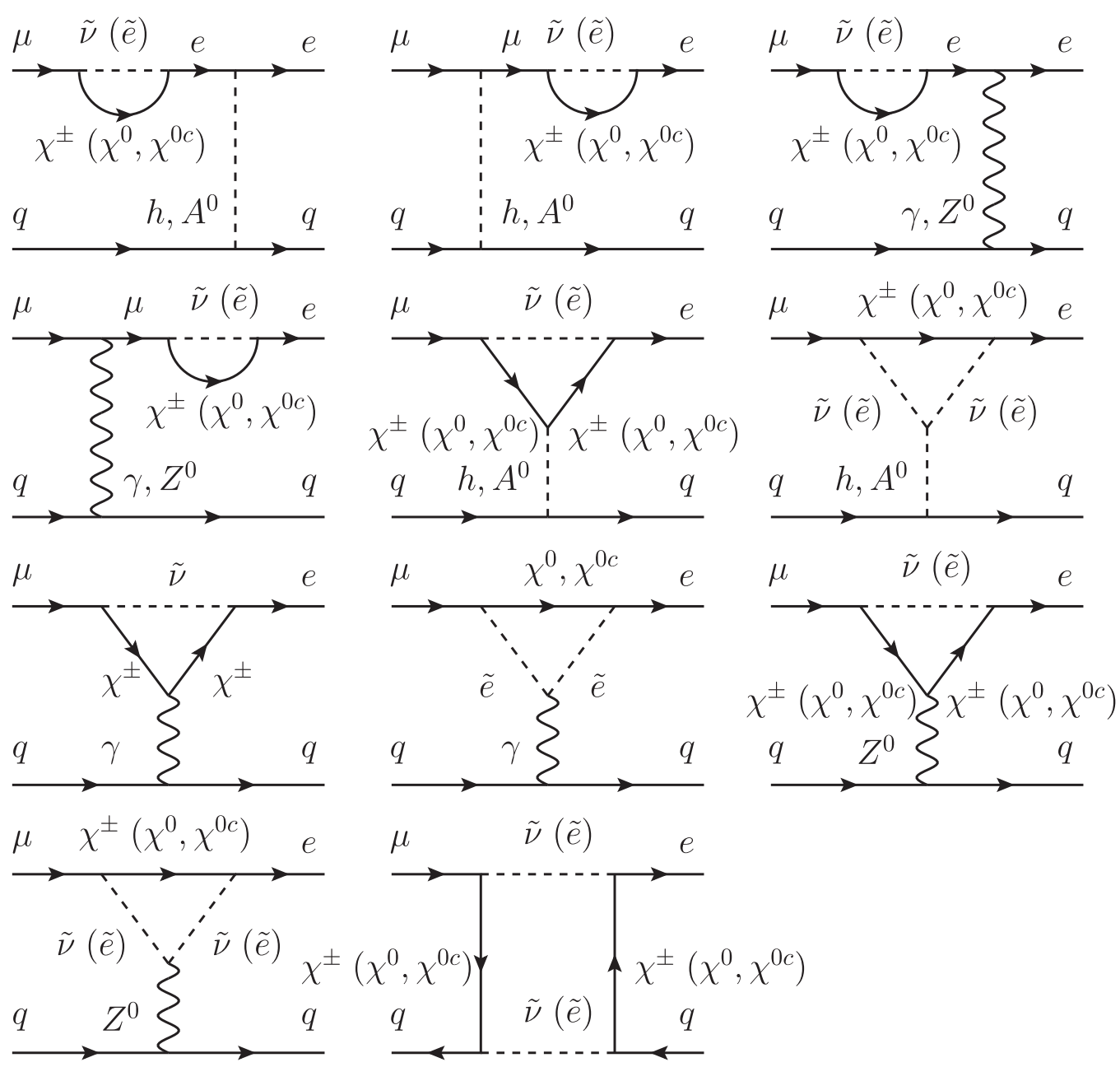

FIG. 1. One loop Feynman diagrams contributing to $\mu$-e conversion in MRSSM.

parameters $S, T$, and $U$. The particle mass spectra are also shown as well as the effective couplings of the lightest Higgs particle to the gauge boson and fermion pairs at leading order. A better agreement with the latest experimental value for the $W$ boson mass has been investigated in Ref. [42]. It combines all numerically relevant contributions that are known in the SM in a consistent way with all MRSSM one-loop corrections. A set of updated benchmark points BMP1 is given in Ref. [42].

In the numerical analysis, we will use two sets of benchmark points which are taken from the above references and display them in Eq. (13) (BMP1) and Eq. (14) (BMP2). All mass parameters in Eq. (13) and Eq. (14) are in $\mathrm{GeV}$ or $\mathrm{GeV}^{2}$,

$$
\begin{aligned}
\tan \beta & =3, \quad B_{\mu}=500^{2}, \quad \lambda_{d}=1.0, \\
\lambda_{u} & =-0.8, \quad \Lambda_{d}=-1.2, \quad \Lambda_{u}=-1.1, \\
M_{D}^{B} & =550, \quad M_{D}^{W}=600, \quad \mu_{d}=\mu_{u}=500, \\
v_{S} & =5.9, \quad v_{T}=-0.33, \\
\left(m_{l}^{2}\right)_{11} & =\left(m_{l}^{2}\right)_{22}=\left(m_{l}^{2}\right)_{33}=\left(m_{r}^{2}\right)_{11}=\left(m_{r}^{2}\right)_{22} \\
& =\left(m_{r}^{2}\right)_{33}=1000^{2}, \\
\left(m_{\tilde{q}}^{2}\right)_{11} & =\left(m_{\tilde{u}}^{2}\right)_{11}=\left(m_{\tilde{d}}^{2}\right)_{11}=\left(m_{\tilde{q}}^{2}\right)_{22}=\left(m_{\tilde{u}}^{2}\right)_{22} \\
& =\left(m_{\tilde{d}}^{2}\right)_{22}=2500^{2}, \\
\left(m_{\tilde{q}}^{2}\right)_{33} & =\left(m_{\tilde{u}}^{2}\right)_{33}=\left(m_{\tilde{d}}^{2}\right)_{33}=1000^{2}, \quad m_{T}=3000, \\
m_{S} & =2000 .
\end{aligned}
$$

TABLE I. Effective atomic charges, nuclear form factors, and capture rates.

\begin{tabular}{lcccccc}
\hline \hline Nucleus ${ }_{Z}^{A} \mathrm{~N}$ & ${ }^{27} \mathrm{Al}$ & ${ }_{22}^{48} \mathrm{Ti}$ & ${ }_{38}^{80} \mathrm{Sb}$ & ${ }_{51}^{121} \mathrm{Sr}$ & ${ }_{79}^{197} \mathrm{Au}$ & ${ }_{82}^{208 \mathrm{~Pb}}$ \\
\hline$Z_{\text {eff }}$ & 11.5 & 17.6 & 25 & 29 & 33.5 & 34 \\
$F_{p}$ & 0.64 & 0.54 & 0.39 & 0.32 & 0.16 & 0.15 \\
$\Gamma_{\text {capt }} \times 10^{18}$ & 0.464079 & 1.70422 & 4.61842 & 6.71711 & 8.59868 & 8.84868 \\
\hline \hline
\end{tabular}




$$
\begin{aligned}
\tan \beta & =10, \quad B_{\mu}=300^{2}, \quad \lambda_{d}=1.1, \\
\lambda_{u} & =-1.1, \quad \Lambda_{d}=-1.0, \quad \Lambda_{u}=-1.0, \\
M_{D}^{B} & =1000, \quad M_{D}^{W}=500, \quad \mu_{d}=\mu_{u}=400, \\
v_{S} & =1.3, \quad v_{T}=-0.19, \\
\left(m_{l}^{2}\right)_{11} & =\left(m_{l}^{2}\right)_{22}=\left(m_{l}^{2}\right)_{33}=\left(m_{r}^{2}\right)_{11}=\left(m_{r}^{2}\right)_{22}=\left(m_{r}^{2}\right)_{33} \\
& =1000^{2}, \\
\left(m_{\tilde{q}}^{2}\right)_{11} & =\left(m_{\tilde{u}}^{2}\right)_{11}=\left(m_{\tilde{d}}^{2}\right)_{11}=\left(m_{\tilde{q}}^{2}\right)_{22}=\left(m_{\tilde{u}}^{2}\right)_{22}=\left(m_{\tilde{d}}^{2}\right)_{22} \\
& =2500^{2}, \\
\left(m_{\tilde{q}}^{2}\right)_{33} & =\left(m_{\tilde{u}}^{2}\right)_{33}=\left(m_{\tilde{d}}^{2}\right)_{33}=1000^{2}, \quad m_{T}=3000, \\
m_{S} & =2000 .
\end{aligned}
$$

In the following numerical analysis, the values in Eqs. (13) and (14) will be used as the default. Note that, the off-diagonal entries of squark mass matrices $m_{\tilde{q}}^{2}, m_{\tilde{u}}^{2}, m_{\tilde{d}}^{2}$ and slepton mass matrices $m_{l}^{2}, m_{r}^{2}$ in Eqs. (13) and (14) are zero, i.e., the flavor mixing of the squark and slepton is absent. Similarly to most supersymmetry models, the LFV processes in the MRSSM originate from the off-diagonal entries of the soft-breaking terms $m_{l}^{2}$ and $m_{r}^{2}$, which are parametrized by mass insertion

$$
\begin{aligned}
& \left(m_{l}^{2}\right)_{I J}=\delta_{l}^{I J} \sqrt{\left(m_{l}^{2}\right)_{I I}\left(m_{l}^{2}\right)_{J J}}, \\
& \left(m_{r}^{2}\right)_{I J}=\delta_{r}^{I J} \sqrt{\left(m_{r}^{2}\right)_{I I}\left(m_{r}^{2}\right)_{J J}},
\end{aligned}
$$

where $I, J=1,2,3$. To decrease the number of free parameters involved in our calculation, we assume that the off-diagonal entries of $m_{l}^{2}$ and $m_{r}^{2}$ in Eq. (15) are equal, i.e., $\delta_{l}^{I J}=\delta_{r}^{I J}=\delta^{I J}$.

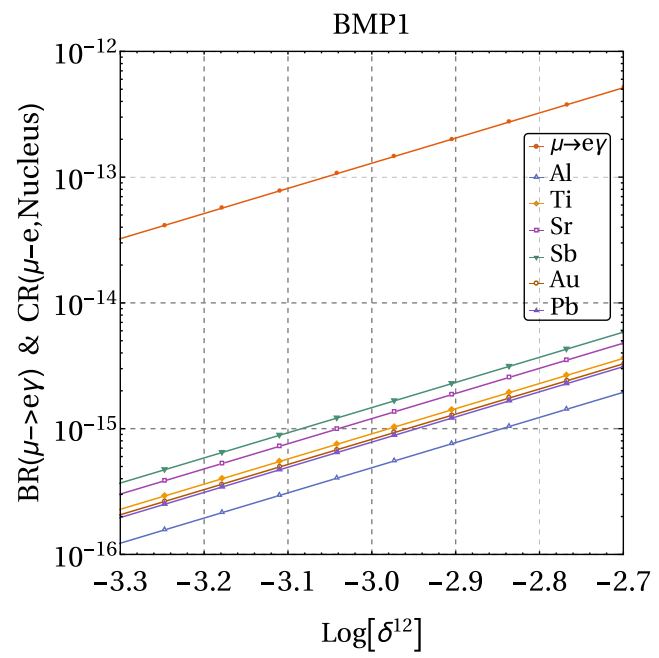

(a)
The experimental limits on LFV decays, such as radiative two body decays $l_{2} \rightarrow l_{1} \gamma$ and leptonic three body decays $l_{2} \rightarrow 3 l_{1}$, can give strong constraints on the parameters $\delta^{I J}$. In the following, we will use LFV decays $\mu \rightarrow e \gamma$ to constrain the parameters $\delta^{12}$ which was discussed in Ref. [54]. It is noted that $\delta^{23}$ and $\delta^{13}$ have been set zero in the following discussion since they have no effect on the predictions of $\mathrm{CR}(\mu-e$, nucleus). The current limit of LFV decays $\mu \rightarrow e \gamma$ is $\operatorname{BR}(\mu \rightarrow e \gamma)<4.2 \times 10^{-13}$ from MEG experiment (MEG) [62] and new sensitivity for this decay channel in the future projects will be $\operatorname{BR}(\mu \rightarrow e \gamma) \sim$ $6 \times 10^{-14}$ from MEG II [63].

In Fig. 2 the predictions for $\mathrm{BR}(\mu \rightarrow e \gamma)$ and $\mathrm{CR}(\mu-e$, nucleus) for $\mathrm{Al}, \mathrm{Ti}, \mathrm{Sr}, \mathrm{Sb}, \mathrm{Au}$, and $\mathrm{Pb}$ are shown as a function of mass insertion parameter $\delta^{12}$ with BMP1 (a) and BMP2 (b). The prediction for $\mathrm{BR}(\mu \rightarrow e \gamma)$ exceeds the future experiment sensitivity at $\delta^{12} \sim 0.001$. In a recent work, Ref. [54], the analytical computation and discussion of $\operatorname{BR}(\mu \rightarrow e \gamma)$ in the MRSSM was performed. The valid region for $\delta^{12}$ calculated in Ref. [54] with the Mathematica package Package- $X$ is compatible with that in this work calculated with SARAH and SPheno. We clearly see that both the predictions for $\operatorname{BR}(\mu \rightarrow e \gamma)$ and $\operatorname{CR}(\mu-e$, nucleus) in nuclei are sensitive to $\delta^{12}$, and they increase along with the increase of $\delta^{12}$ which has the same behavior as those in most SUSY models (see e.g., [64]). At $\delta^{12} \sim 0.001$, the prediction on $\operatorname{BR}(\mu \rightarrow e \gamma)$ is very close to the current experimental limit, and the predictions on $\mathrm{CR}(\mu-e$, nucleus $)$ are around $10^{-15}-10^{-16}$ which are 2 orders of magnitude below current experimental limits. The predicted $\mathrm{CR}(\mu-e, \mathrm{Ti})$ is around $10^{-15}$ and this is 3 orders of magnitude above future experimental sensitivity [4]. The predicted $\operatorname{CR}(\mu-e, \mathrm{Al})$ is around $10^{-16}$ and this is in the region of the future experimental sensitivity [5,6]. In Fig. 2,

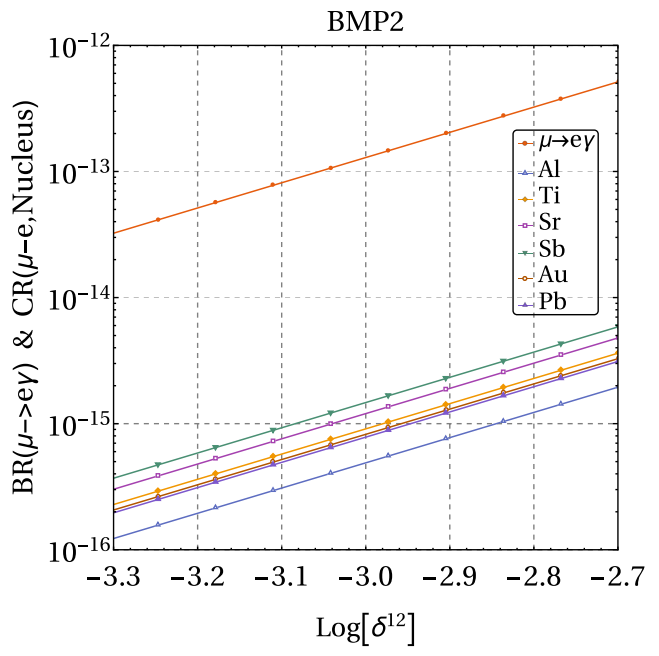

(b)

FIG. 2. Dependence of $\operatorname{BR}(\mu \rightarrow e \gamma)$ and $\operatorname{CR}(\mu-e$, nucleus $)$ on the logarithm of mass insertion parameter $\delta^{12}$ to the base 10 . All other parameters are set to the values of benchmark points BMP1 (a) and BMP2 (b). 
the predicted $\mathrm{CR}(\mu-e$, nucleus) with $\mathrm{BMP} 1$ are higher than those with BMP2, and this is because of a larger $\tan \beta$ and a smaller $M_{D}^{W}$ in BMP2 than those in BMP1.

As shown in Fig. 2, the predicted $\mathrm{CR}(\mu-e$, nucleus) in various nuclei are very close to each other. A lower $\mathrm{CR}(\mu-e, \mathrm{Al})$ together with an upper $\mathrm{CR}(\mu-e, \mathrm{Sb})$ is predicted within the same parameter space for BMP1 and BMP2, respectively. It is compatible with the result in Ref. [65] which indicates the $\mathrm{CR}(\mu-e$, nucleus) increases for a light nucleus up to the atomic number $Z<30$, is largest for $Z=30-60$, and becomes smaller for a heavy nucleus with $Z>60$. In the following we will display the predicted $\operatorname{CR}(\mu-e$, nucleus $)$ in one nucleus with $\delta^{12}=$ 0.001 in each plot and the predicted $\operatorname{BR}(\mu \rightarrow e \gamma)$ for all points in each plot satisfy the current experimental bound.

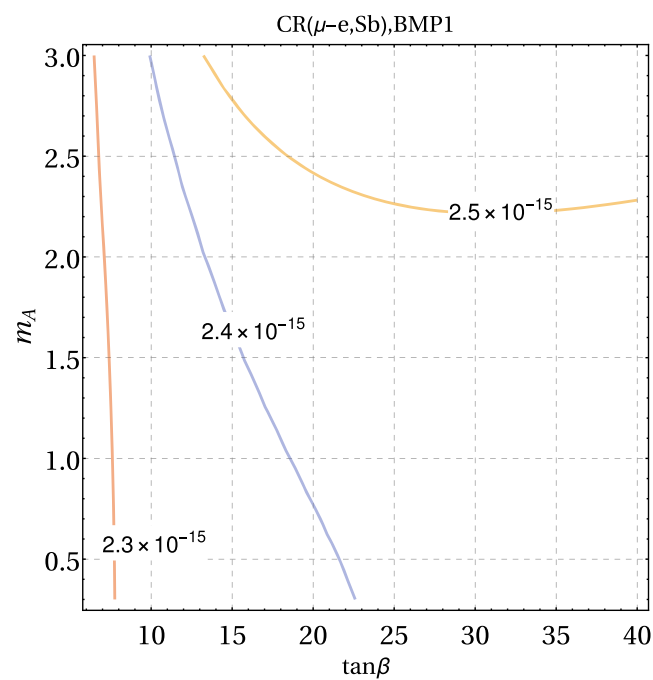

(a)

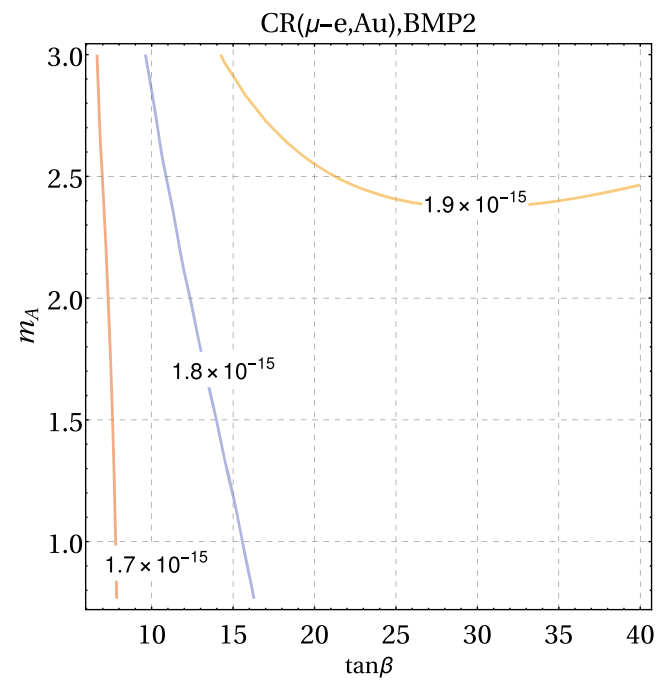

(c)
In Fig. 3 the predictions for $\mathrm{CR}(\mu-e$, nucleus $)$ are shown as a function of $\tan \beta$ and $m_{A}$. This is realized by varying parameter $B_{\mu}$ which is related to $m_{A}$ through the equation $m_{A}^{2}=\frac{2 B_{\mu}}{\sin 2 \beta}$. We clearly see that the predictions for $\mathrm{CR}(\mu-e$, nucleus $)$ grow as $\tan \beta$ or $m_{A}$ grows. The predictions for $\mathrm{CR}(\mu-e$, nucleus) in nuclei are not sensitive to $\tan \beta$ or $m_{A}$ and take values in a narrow region. This is a striking difference to some SUSY models $[28,29,31,43]$. Due to the existence of the transition from $d$-Higgsino to $u$-Higgsino in the MSSM, which is governed by the $\mu$ term, the well-known $\tan \beta$ enhancement is possible. A well-established way to understand the $\tan \beta$ enhancement is provided by mass-insertion diagrams involving insertions of the $\mu$ parameter and Majorana gaugino masses. However, the $\mu$ term and Majorana

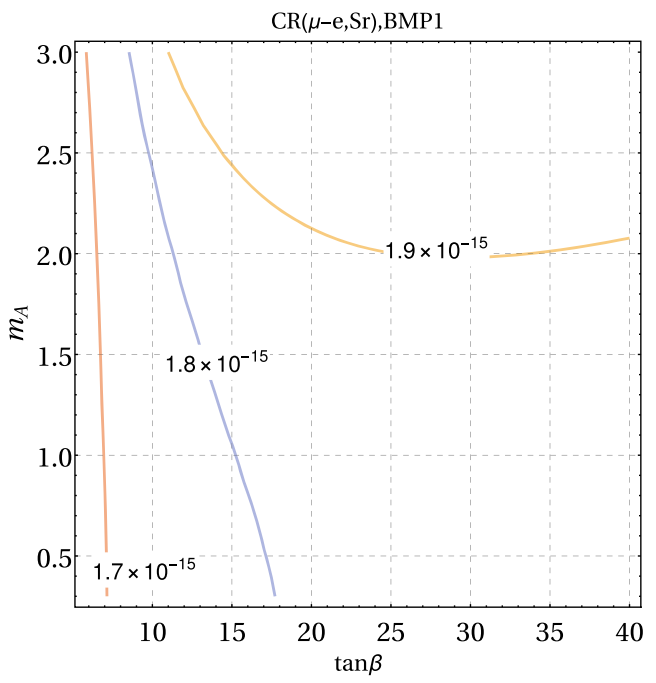

(b)

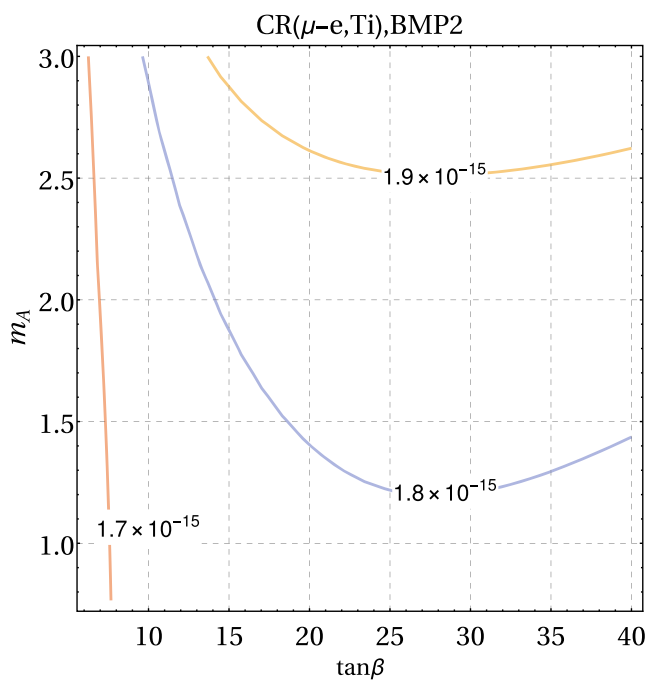

(d)

FIG. 3. Dependence of $\mathrm{CR}\left(\mu-e\right.$, nucleus) on $\tan \beta$ and $m_{A}$. All other parameters are set to the values of benchmark points BMP1 (top row) and BMP2 (bottom row). 
gaugino masses are forbidden in the MRSSM and this leads to the result that $\mathrm{CR}(\mu-e$, nucleus) are not enhanced by $\tan \beta$.

The predictions for $\mathrm{CR}(\mu-e$, nucleus $)$ in $\mathrm{Al}$ and $\mathrm{Sb}$ are shown in Fig. 4 as a function of the squark mass parameter $\mathrm{mQ}$ and the slepton mass parameter $\mathrm{mL}$. Here, $\mathrm{mL}=$ $\sqrt{\left(m_{l}^{2}\right)_{11}}=\sqrt{\left(m_{l}^{2}\right)_{22}}=\sqrt{\left(m_{l}^{2}\right)_{33}}=\sqrt{\left(m_{r}^{2}\right)_{11}}=\sqrt{\left(m_{r}^{2}\right)_{22}}=$ $\sqrt{\left(m_{r}^{2}\right)_{33}}$ and $\mathrm{mQ}=\sqrt{\left(m_{\tilde{q}}^{2}\right)_{11}}=\sqrt{\left(m_{\tilde{u}}^{2}\right)_{11}}=\sqrt{\left(m_{\tilde{d}}^{2}\right)_{11}}=$ $\sqrt{\left(m_{\tilde{q}}^{2}\right)_{22}}=\sqrt{\left(m_{\tilde{u}}^{2}\right)_{22}}=\sqrt{\left(m_{\tilde{d}}^{2}\right)_{22}}=\sqrt{\left(m_{\tilde{q}}^{2}\right)_{33}}=\sqrt{\left(m_{\tilde{u}}^{2}\right)_{33}}=$ $\sqrt{\left(m_{\tilde{d}}^{2}\right)_{33}}$. We clearly see that the predictions for $\mathrm{CR}(\mu-e$, nucleus $)$ in nuclei are sensitive to $\mathrm{mQ}$ and $\mathrm{mL}$, and they decrease along with the increase of $\mathrm{mQ}$ and $\mathrm{mL}$ which is described as a baseline behavior as those in Refs. [43,64]. In a wide region of $\mathrm{mL}$ and $\mathrm{mQ}$, the predicted $\mathrm{CR}(\mu-e, \mathrm{Ti})$ is around $10^{-16}$ and this is still 2 orders of magnitude above future experimental sensitivity [4], and the predicted $\mathrm{CR}(\mu-e, \mathrm{Al})$ is below $10^{-16}$ and this is in the region of the future experimental sensitivity $[5,6]$. Only the contribution from box diagrams for $\mathrm{CR}(\mu-e$, nucleus) depend on the squark masses. This means the contribution from box diagrams is comparable with other diagrams.

It is noted that the predictions for $\mathrm{CR}(\mu-e$, nucleus $)$ in nuclei show a weak dependence on the wino-triplino mass parameter $M_{D}^{W}$, and they decrease slowly along with the

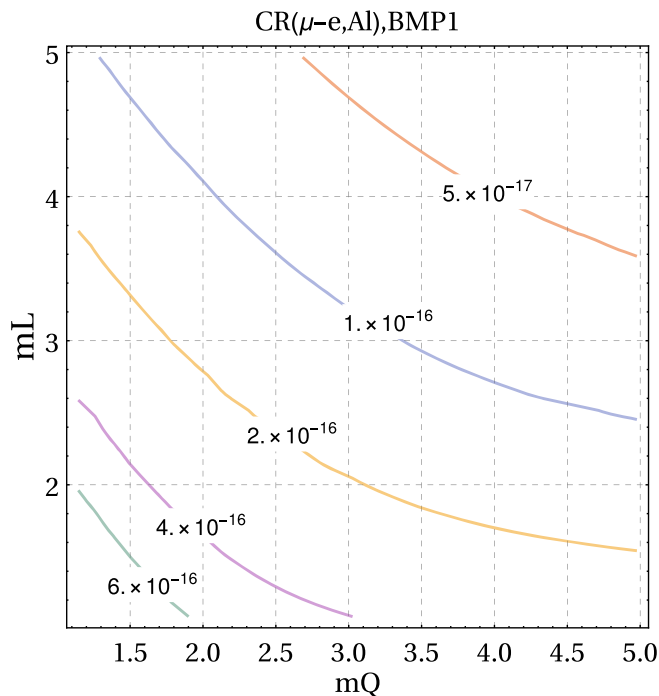

(a)

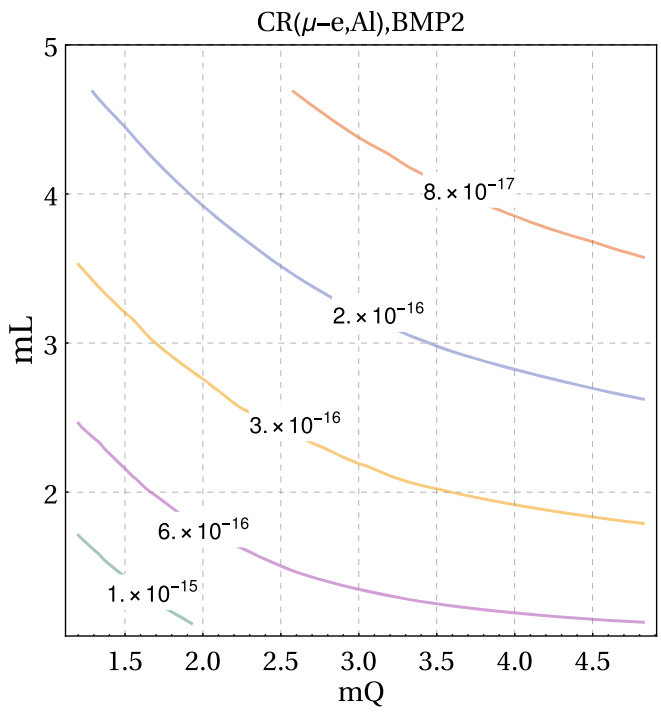

(c)

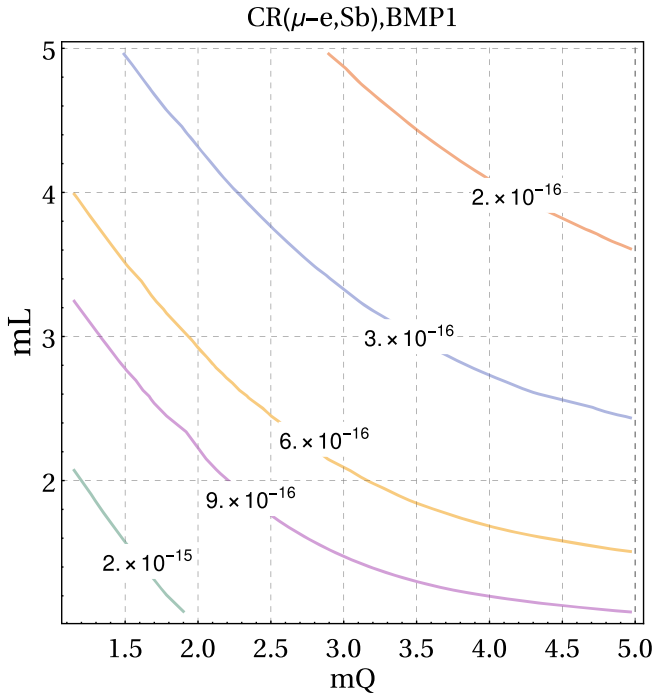

(b)

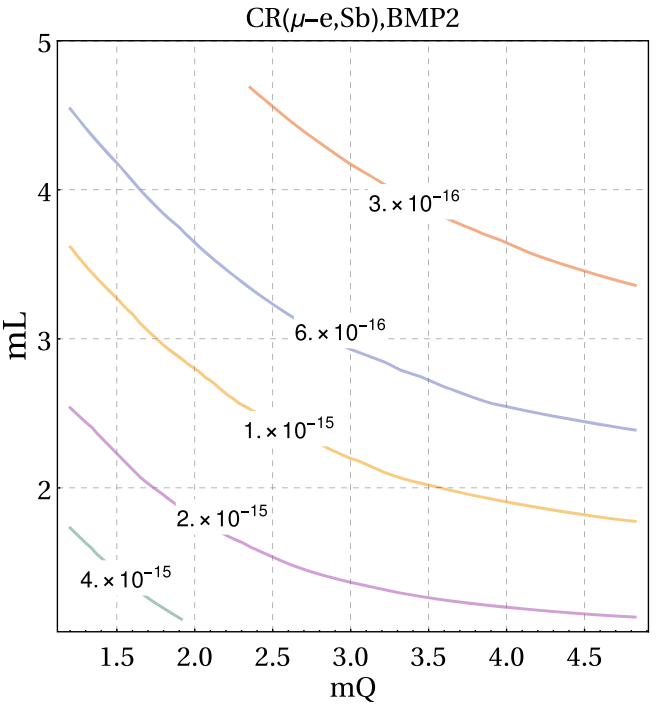

(d)

FIG. 4. Dependence of $\mathrm{CR}(\mu-e$, nucleus) on squark mass parameter $\mathrm{mQ}$ and slepton mass parameter mL. All other parameters are set to the values of benchmark points BMP1 (top row) and BMP2 (bottom row), and all mass parameters are in TeV. 


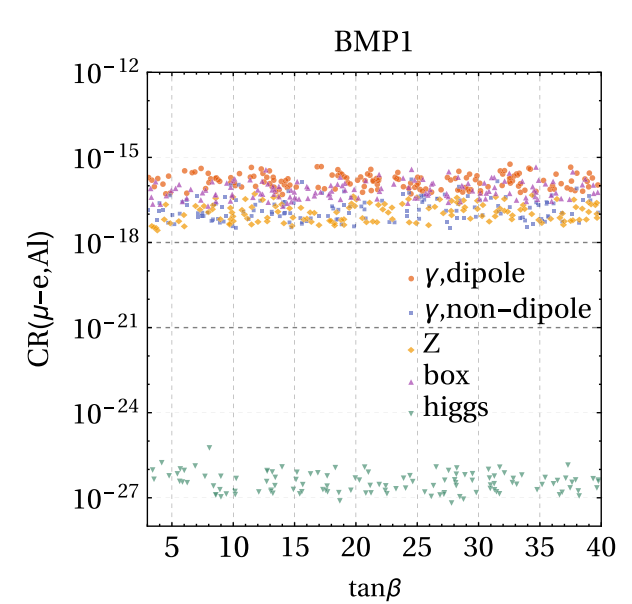

(a)

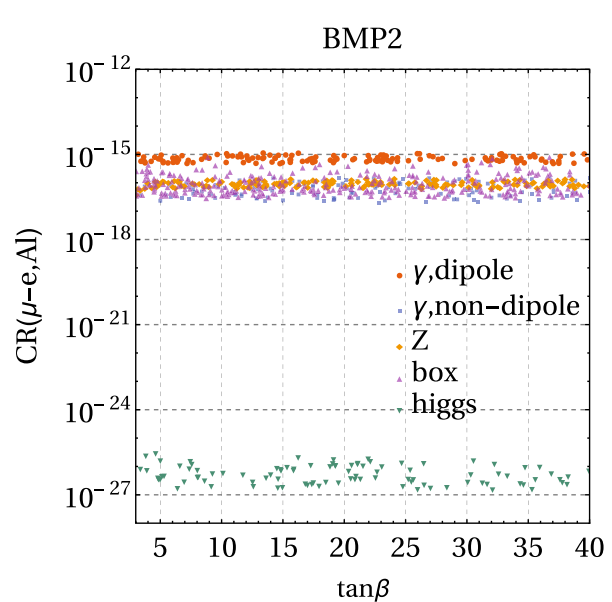

(b)

FIG. 5. Contributions from $\gamma$ dipole (blue dot), $\gamma$ nondipole (orange square), $Z$ penguins (green diamond), box diagrams (brown triangle), and Higgs penguins (purple inverted triangle) and to $\mathrm{CR}(\mu-e, \mathrm{Al}$ ) as a function of $\tan \beta$. All other parameters are set to the values of benchmark points BMP1 (a) and BMP2 (b).

increase of $M_{D}^{W}$. However, the valid region of $M_{D}^{W}$ is constrained by the boundary conditions at the unification scale, and unphysical masses of neutral Higgs and charged Higgs are obtained when $M_{D}^{W}$ above several TeV. We are also interested in the effects from other parameters on the predictions of $\mathrm{CR}(\mu-e$, nucleus $)$ in the MRSSM such as $M_{D}^{B}, \lambda_{d}, \lambda_{u}, \Lambda_{d}, \Lambda_{u}, \mu_{d}$, and $\mu_{u}$. By scanning over these parameters, the results show these parameters are also constrained in a narrow band and the predictions for $\mathrm{CR}(\mu-e$, nucleus) take values along a narrow region.

In Fig. 5, we show the predictions on $\mathrm{CR}(\mu-e, \mathrm{Al})$ as a function of $\tan \beta$ with benchmark points BMP1 (a) and BMP2 (b) but independently considering the contributions from each diagram, and the values of $\mathrm{CR}(\mu-e, \mathrm{Al})$ are given by only the listed contribution with all others set to zero. The range of input parameters for the numerical scan is given in Eq. (16). All other parameters are set to the values of benchmark points BMP1 (a) and BMP2 (b),

$$
\begin{aligned}
3 & <\tan \beta<40 ; \quad 300 \mathrm{GeV}<M_{D}^{W}, \quad M_{D}^{B}<1000 \mathrm{GeV} \\
9 & \times 10^{4} \mathrm{GeV}^{2}<B_{\mu}<10^{6} \mathrm{GeV}^{2} ; \\
m Q & <3000 \mathrm{GeV} .
\end{aligned}
$$

We observe that the dipole contributions $A_{2}^{L / R}$ from $\gamma$ penguins dominate the predictions on $\mathrm{CR}(\mu-e, \mathrm{Al})$ similar to the case in some SUSY models (see e.g., [30]). The contributions from Higgs penguins is negligible. In the supersymmetric seesaw model, LFV in the Higgs coupling originates from the nonholomorphic correction to the Yukawa interactions of the charged leptons [66] which involves the gaugino and Higgsino mass parameters. However, these parameters are absent in the MRSSM. Thus the Higgs-exchange diagrams play a different role in the MRSSM from that in other SUSY models [25]. The predicted $\mathrm{CR}(\mu-e, \mathrm{Al})$ of Higgs penguins would be even smaller when $M_{D}^{W}$ is close to the boundary conditions.

The nondipole contributions $A_{1}^{L / R}$ from $\gamma$ penguins and the contributions from $Z$ penguins and box diagrams are less dominant in a large parameter region. In the MSSM, for a small ratio of wino mass to slepton mass, the predicted $\mathrm{CR}(\mu-e$, nucleus $)$ is dominated by the dipole contributions [67]. There is a simple relation between the $\mathrm{CR}(\mu-e$, nucleus) and $\operatorname{BR}(\mu \rightarrow e \gamma)$. Given the future experimental improvements on measuring both $\mathrm{CR}(\mu-e$, nucleus) and $\mathrm{BR}(\mu \rightarrow e \gamma)$, $\mu$-e conversion can impose limits on LFV insertions comparable to those from $\mu \rightarrow e \gamma$. The nondipole contributions from $\gamma / Z$ penguins dominate the predictions on $\mathrm{CR}(\mu-e$, nucleus) for a small ratio of a common mass to slepton mass. In the MRSSM, the predicted $\mathrm{CR}(\mu-e, \mathrm{Al})$ of box diagrams can reach similar magnitudes as those from the dipole contributions of $\gamma$ penguins when $\mathrm{mQ} \sim 1 \mathrm{TeV}$, or similar magnitudes as those from $Z$ penguins or nondipole contributions of $\gamma$ penguins when $\mathrm{mQ} \sim 3 \mathrm{TeV}$. Thus, by considering the contributions form nondipole diagrams, the predicted $\mathrm{CR}(\mu-e$, nucleus) could be increased even larger than the predicted $\mathrm{BR}(\mu \rightarrow e \gamma)$ (see e.g., [27]). This makes it possible to observe $\mu-e$ conversion in experiment while no signals of $\mu \rightarrow e \gamma$ or $\mu \rightarrow 3 e$ are obtained [26].

\section{CONCLUSIONS}

In this work, taking into account the constraints from $\mu \rightarrow e \gamma$ on the parameter space, we analyze the LFV process $\mathrm{CR}(\mu-e$, nucleus $)$ in the framework of the minimal R-symmetric supersymmetric standard model. In this model, R-symmetry forbids Majorana gaugino masses, $\mu$ term, $A$ terms, and all left-right squark and slepton mass 
mixings. Due to the absence of a $\mu$ term and Majorana gaugino masses, the predictions for $\mathrm{CR}(\mu-e$, nucleus $)$ are not enhanced by $\tan \beta$. This is the main difference to the MSSM.

Besides the constraints considered in Sec. III, restrictions arising from the ATLAS and CMS searches for heavy Higgs bosons in the ditau channel should also be considered. The effect of this collider search is to impose an upper limit on $\tan \beta$. The latest search for a scalar or pseudoscalar decaying to a pair of taus with simplified exclusion likelihoods has been released by ATLAS by using $139 \mathrm{fb}^{-1}$ of integrated luminosity at $13 \mathrm{TeV}$ [68]. This model independent likelihood has been properly implemented in the new version of HiggsBounds-5 [69]. We would like to postpone this work to our next article which analyzes the LFV decays of SM-like Higgs in the MRSSM. The change of $m_{A}$ has a small effect on predictions of $\operatorname{BR}(\mu \rightarrow e \gamma)$ and $\mathrm{CR}(\mu-e$, nucleus $)$ for small $\tan \beta$ as shown in Fig. 3. For large $\tan \beta$, the effect of $m_{A}$ on the predictions for $\mathrm{CR}(\mu-e$, nucleus $)$ is also small and the latter take values along a narrow region. In Ref. [68] values of $\tan \beta>8$ and $\tan \beta>21$ are excluded at the $95 \%$ confidence level for $m_{A}=1.0 \mathrm{TeV}$ and $m_{A}=1.5 \mathrm{TeV}$ in the $M_{h}^{125}$ scenario of the MSSM, respectively. In the MRSSM, corresponding to $\tan \beta=3$ and $\tan \beta=10$, the default values of $m_{A}$ are
$m_{A}=0.912 \mathrm{TeV}$ and $m_{A}=0.953 \mathrm{TeV}$ for BMP1 in Eq. (13) and BMP2 in Eq. (14), respectively.

In the MRSSM, the theoretical predictions on $\mathrm{CR}(\mu-e$, nucleus) mainly depend on the mass insertion $\delta^{12}$. The predictions on $\mathrm{CR}\left(\mu-e\right.$, nucleus) would be zero if $\delta^{12}=0$ is assumed. Taking into account the experimental bounds on radiative decays $\mu \rightarrow e \gamma$, the values of $\delta^{12}$ are constrained around 0.001 . Assuming $\delta^{12}=0.001$ and other parameter settings in Eq. (13), the predictions on $\mathrm{CR}(\mu-e$, nucleus) are at the level of $\mathcal{O}\left(10^{-15}-10^{-16}\right)$, which are 2 or 3 orders of magnitude above the future experimental prospects for an $\mathrm{Al}$ or $\mathrm{Ti}$ target. Thus, the LFV processes $\mu$-e conversion in $\mathrm{Al}$ and $\mathrm{Ti}$ are very promising to be observed in near future experiments.

\section{ACKNOWLEDGMENTS}

This work has been supported partly by the National Natural Science Foundation of China (NNSFC) under Grants No. 11905002, No. 11805140, and No. 11705045 , the Scientific Research Foundation of the Higher Education Institutions of Hebei Province under Grant No. BJ2019210, the Foundation of Baoding University under Grant No. 2018Z01, and the youth top-notch talent support program of the Hebei Province.
[1] S. Ahmad et al., Phys. Rev. Lett. 59, 970 (1987).

[2] W. Bertl, Eur. Phys. J. C 47, 337 (2006).

[3] D. Aoki, AIP Conf. Proc. 1441, 599 (2012).

[4] R. J. Barlow, Nucl. Phys. B, Proc. Suppl. 218, 44 (2011).

[5] L. Bartoszek et al., arXiv:1501.05241.

[6] A. Kurup, Nucl. Phys. B, Proc. Suppl. 218, 38 (2011).

[7] Riazuddin, R. E. Marshak, and R. N. Mohapatra, Phys. Rev. D 24, 1310 (1981).

[8] L. N. Chang, D. Ng, and J. N. Ng, Phys. Rev. D 50, 4589 (1994).

[9] A. Ioannisian and A. Pilaftsis, Phys. Rev. D 62, 066001 (2000).

[10] A. Pilaftsis and T. E. J. Underwood, Phys. Rev. D 72, 113001 (2005).

[11] F. Deppisch, T. S. Kosmas, and J. W. F. Valle, Nucl. Phys. B752, 80 (2006).

[12] A. Ilakovac and A. Pilaftsis, Phys. Rev. D 80, 091902 (2009).

[13] F. F. Deppisch and A. Pilaftsis, Phys. Rev. D 83, 076007 (2011).

[14] M. Raidal and A. Santamaria, Phys. Lett. B 421, 250 (1998).

[15] E. Ma, M. Raidal, and U. Sarkar, Nucl. Phys. B615, 313 (2001).

[16] D. N. Dinh, A. Ibarra, E. Molinaro, and S. T. Petcov, J. High Energy Phys. 08 (2012) 125; 09 (2013) 23.
[17] K.-S. Sun, T.-F. Feng, G.-H. Luo, X.-Y. Yang, and J.-B. Chen, Mod. Phys. Lett. A 28, 1350151 (2013).

[18] A. Abada, C. Biggio, F. Bonnet, M. B. Gavela, and T. Hambye, Phys. Rev. D 78, 033007 (2008).

[19] G.-J. Ding and M.-L. Yan, Phys. Rev. D 77, 014005 (2008).

[20] K.-S. Sun, T.-F. Feng, L.-N. Kou, F. Sun, T.-J. Gao, and H.-B. Zhang, Mod. Phys. Lett. A 27, 1250172 (2012).

[21] M. Blanke, A. J. Buras, B. Duling, A. Poschenrieder, and C. Tarantino, J. High Energy Phys. 05 (2007) 013.

[22] F. D. Aguila, J. I. Illana, and M. D. Jenkins, J. High Energy Phys. 03 (2011) 080.

[23] C. Bonilla, M. E. Krauss, T. Opferkuch, and W. Porod, J. High Energy Phys. 03 (2017) 027.

[24] D. T. Huong, D. N. Dinh, L. D. Thien, and P. V. Dong, J. High Energy Phys. 08 (2019) 051.

[25] J. Hisano, S. Sugiyama, M. Yamanaka, and M. J. S. Yang, Phys. Lett. B 694, 380 (2011).

[26] J. Sato and M. Yamanaka, Phys. Rev. D 91, 055018 (2015).

[27] A. Ilakovac, A. Pilaftsis, and L. Popov, Phys. Rev. D 87, 053014 (2013).

[28] T. Guo, S.-M. Zhao, X.-X. Dong, C.-G. Duan, and T.-F. Feng, Eur. Phys. J. C 78, 925 (2018).

[29] X.-X. Dong, S.-M. Zhao, H.-B. Zhang, and T.-F. Feng, Eur. Phys. J. C 79, 17 (2019).

[30] E. Arganda, M. J. Herrero, and A. M. Teixeira, J. High Energy Phys. 10 (2007) 104. 
[31] H.-B. Zhang, T.-F. Feng, G.-H. Luo, Z.-F. Ge, and S.-M. Zhao, J. High Energy Phys. 07 (2013) 069.

[32] A. Crivellin, M. Hoferichter, and M. Procura, Phys. Rev. D 89, 093024 (2014).

[33] L. Calibbi and G. Signorelli, Riv. Nuovo Cimento 41, 71 (2018).

[34] R. H. Bernstein and P. S. Cooper, Phys. Rep. 532, 27 (2013).

[35] M. Lindner, M. Platscher, and F. S. Queiroz, Phys. Rep. 731, 1 (2018).

[36] G. D. Kribs, E. Poppitz, and N. Weiner, Phys. Rev. D 78, 055010 (2008).

[37] P. Diessner and W. Kotlarski, Proc. Sci., CORFU2014 (2015) 079.

[38] P. Diessner, J. Kalinowski, W. Kotlarski, and D. Stöckinger, Adv. High Energy Phys. 2015, 1 (2015).

[39] P. Diessner, J. Kalinowski, W. Kotlarski, and D. Stöckinger, J. High Energy Phys. 12 (2014) 124.

[40] P. Diessner, W. Kotlarski, S. Liebschner, and D. Stöckinger, J. High Energy Phys. 10 (2017) 142.

[41] P. Diessner, J. Kalinowski, W. Kotlarski, and D. Stöckinger, J. High Energy Phys. 03 (2016) 007.

[42] P. Diessner and G. Weiglein, J. High Energy Phys. 07 (2019) 011.

[43] W. Kotlarski, D. Stöckinger, and H. Stöckinger-Kim, J. High Energy Phys. 08 (2019) 082.

[44] A. Kumar, D. Tucker-Smith, and N. Weiner, J. High Energy Phys. 09 (2010) 111.

[45] A. E. Blechman, Mod. Phys. Lett. A 24, 633 (2009).

[46] G. D. Kribs, A. Martin, and T. S. Roy, J. High Energy Phys. 06 (2009) 042.

[47] C. Frugiuele and T. Gregoire, Phys. Rev. D 85, 015016 (2012).

[48] J. Kalinowski, Acta Phys. Pol. B 47, 203 (2016).

[49] S. Chakraborty, A. Chakraborty, and S. Raychaudhuri, Phys. Rev. D 94, 035014 (2016).
[50] J. Braathen, M. D. Goodsell, and P. Slavich, J. High Energy Phys. 09 (2016) 045.

[51] P. Athron, J.-H. Park, T. Steudtner, D. Stöckinger, and A. Voigt, J. High Energy Phys. 01 (2017) 079.

[52] C. Alvarado, A. Delgado, and A. Martin, Phys. Rev. D 97, 115044 (2018).

[53] K.-S. Sun, J.-B. Chen, X.-Y. Yang, and H.-B. Zhang, Mod. Phys. Lett. A 34, 1950058 (2019).

[54] K.-S. Sun, J.-B. Chen, X.-Y. Yang, and S.-K. Cui, Chin. Phys. C 43, 043101 (2019).

[55] K.-S. Sun, J.-B. Chen, H.-B. Zhang, and S.-K. Cui, Mod. Phys. Lett. A 35, 1950359 (2020).

[56] F. Staub, arXiv:0806.0538.

[57] F. Staub, Comput. Phys. Commun. 184, 1792 (2013).

[58] F. Staub, Comput. Phys. Commun. 185, 1773 (2014).

[59] W. Porod, F. Staub, and A. Vicente, Eur. Phys. J. C 74, 2992 (2014).

[60] W. Porod, Comput. Phys. Commun. 153, 275 (2003).

[61] W. Porod and F. Staub, Comput. Phys. Commun. 183, 2458 (2012).

[62] A. M. Baldini et al. (MEG Collaboration), Eur. Phys. J. C 76, 434 (2016).

[63] A. M. Baldini et al. (MEG Collaboration), Eur. Phys. J. C 78, 380 (2018).

[64] K.-S. Sun, T.-F. Feng, T.-J. Gao, and S.-M. Zhao, Nucl. Phys. B865, 486 (2012).

[65] R. Kitano, M. Koike, and Y. Okada, Phys. Rev. D 66, 096002 (2002); 76, 059902(E) (2007).

[66] R. Kitano, M. Koike, S. Komine, and Y. Okada, Phys. Lett. B 575, 300 (2003).

[67] S. A. R. Ellis and A. Pierce, Phys. Rev. D 94, 015014 (2016).

[68] ATLAS Collaboration, Phys. Rev. Lett. 125, 051801 (2020).

[69] P. Bechtle, D. Dercks, S. Heinemeyer, T. Klingl, T. Stefaniak, G. Weiglein et al., arXiv:2006.06007. 